Philosophie ANTIQUE

\section{Philosophie antique}

Problèmes, Renaissances, Usages

17 | 2017

Platon et la politique

\title{
Bulletin bibliographique
}

\section{(2) OpenEdition \\ 1 Journals}

Édition électronique

URL : https://journals.openedition.org/philosant/325

DOI : 10.4000/philosant.325

ISSN : 2648-2789

Éditeur

Éditions Vrin

\section{Édition imprimée}

Date de publication : 1 novembre 2017

Pagination : 231-233

ISBN : 978-2-7574-1807-9

ISSN : 1634-4561

\section{Référence électronique}

«Bulletin bibliographique », Philosophie antique [En ligne], 17 | 2017, mis en ligne le 01 novembre 2018, consulté le 10 décembre 2022. URL : http://journals.openedition.org/philosant/325 ; DOI : https://

doi.org/10.4000/philosant.325

\section{(c) (i) (9)}

Creative Commons - Attribution - Pas d'Utilisation Commerciale - Pas de Modification 4.0 International - CC BY-NC-ND 4.0

https://creativecommons.org/licenses/by-nc-nd/4.0/ 


\section{Bulletin Bibliographique}

\section{Éditions, traductions et commentaires}

- Plato, Theaetetus and Sophist, edited by Christopher Rowe, Cambridge, Cambridge University Press, 2015, ISBN : 978-1-10-701483-1.

Traduction nouvelle précédée d'une introduction, insistant sur la continuité entre les deux dialogues et leurs relations avec certains autres, notamment La République.

- Priscian, Answers to King Khosroes of Persia, translated by Pamela Huby, Sten Ebbesen, David Langslow, Donald Russell, Carlos Steel and Malcolm Wilson, Introduction by Richard Sorabji, Londres, Bloomsbury, 2016 (Ancient Commentators on Aristotle), ISBN HB : 978-1-47258-413-7 ; ePub : 978-1-47258-41 ; ePDF : 978-147258-415-1.

Les Solutiones ad Chosroem de Priscien de Lydie sont un condensé des réponses des philosophes grecs aux questions posées par le roi Chosroès aux philosophes néoplatoniciens qu'il avait accueillis après la fermeture de l'Académie par Justinien. Elles ne sont connues que dans une traduction latine tardive, souvent attribuée à Jean Scot Érigène, ce que met en doute R. Sorabji au vu de la faible connaissance, tant du grec que des questions traitées par Priscien, qui semble avoir été celle du traducteur. La présente traduction a été obtenue à partir d'une reconstitution de ce que pouvait être le sens du texte grec original, par recours aux sources utilisées par Priscien ou par des tentatives de rétroversion du latin vers le grec.

- Plotin, Traité 20 (I, 3) Sur la Dialectique, Introduction, traduction, commentaire et notes par Jean-Baptiste Gourinat, Paris, Librairie philosophique J. Vrin, 2016 (Les Écrits de Plotin), ISBN 978-2-7116-2694-6.

Ce volume inaugure la reprise à la Librairie Vrin de la collection « Les Écrits de Plotin », fondée naguère par Pierre Hadot aux Éditions du Cerf. Seul le choix d'une nouvelle couverture signale une éventuelle volonté de rajeunissement. La composition du volume, elle, est identique à celle des volumes parus au Cerf, marquant ainsi la continuité de la collection : une introduction détaillant la structure et les thèmes du traité, suivie de la traduction de celui-ci équipée d'intertitres de différents niveaux dus au traducteur, destinés à faciliter au lecteur l'intelligence du texte, puis un abondant commentaire reprenant point par point le déroulement du traité.

- Premiers écrits chrétiens. Textes traduits du grec ancien, du latin, de l'arabe, de l'arménien, de l'hébreu, du slavon et du syriaque, sous la direction de Bernard Pouderon, Jean-Marie Salamito et Vincent Zarini, Paris, Gallimard, 2016 (Bibliothèque de la Pléiade, 617), ISBN 978-2-0701-3486-1.

Rédigés entre la fin du Ir siècle apr. J.-C. et le début du III ${ }^{\mathrm{c}}$, les textes rassemblés dans ce volume documentent l'autodéfinition du christianisme, à travers usages liturgiques, constitution d'un canon des Écritures et d'un corps de doctrines.

\section{Études}

- Livio Rossetti, La Filosofia non nasce con Talete e nemmeno con Socrate. Bologne, Diogene Multimedia, 2015 (Saggi), ISBN 978-8-89912-639-1.

- Francesco Aronadio, L'aisthesis e le strategie argomentative di Platone nel Teeteto, Naples, Bibliopolis, 2016 (Elenchos, 63), ISBN 978-8-87088-640-5. 


\section{Recueils}

- Richard Sorabji (éd.), Aristotle Re-Interpreted: New Findings on Seven Hundred Years of the Ancient Commentators, Londres, Bloomsbury, 2016 (Ancient Commentators on Aristotle), ISBN HB : 978-1-47259-656-7 ; ePub : 978-1-47259-6574 ; ePDF : 978-1-47259-658-1.

Après une substantielle introduction de R. Sorabji ( $\ll$ Seven Hundred Years of Commentary and Sixth Century Diffusion to Other Cultures », p. 1-80), ce volume comprend 23 contributions, datées de 1994 à 2015. Certaines sont des versions remaniées d'articles antérieurs, cinq sont inédites.

À cette occasion, R. Sorabji publie chez le même éditeur une seconde édition du recueil paru en 1990 chez Duckworth : Aristotle Transformed: The Ancient Commentators and Their Influence, ISBN HB : 978-1-47258-907-1 ; ePDF : 978-1-47258-909-5 ; ePDF : 978-1-47258-908-8. Cette édition ne diffère de la précédente que par une introduction rédigée spécialement par R. Sorabji («Introduction to the Second Edition », p. xii-xlvii), dans laquelle il actualise certaines contributions en signalant les évolutions de la recherche intervenues entre-temps.

- Rodolphe Calin, Jean-Luc Périllié, Olivier Tinland (éd.), Platon et la philosophie française contemporaine. Enjeux philologiques, historiques et philosophiques, Bruxelles, Éditions Ousia, 2016 (Cahiers de philosophie ancienne, 24), ISBN 978-2-87060-182-2. Onze contributions issues du colloque tenu à l'Université Paul-Valéry Montpellier 3 du 13 au 15 novembre 2014.

- Étienne Helmer (éd.), Richesse et pauvreté chez les philosophes de l'Antiquité, Paris, Librairie philosophique J. Vrin, 2016 (Tradition de la pensée classique), ISBN 978-27116-2685-4.

Recueil de douze études recensant les façons dont les philosophes, d'Antiphon à Augustin, ont pensé richesse et pauvreté et comment ils en ont analysé les conséquences morales, sociales, économiques et politiques.

- Fulvia de Luise (éd.), Legitimazione del potere, autorità della legge : un dibattito antico, Trente, Università degli Studi di Trento, Dipartimento di Lettere e Filosofia, 2016 (Studi e Ricerche, 10), ISBN 978-88-8443-636-8.

Préface de F. de Luise, Introduction de M. Vegetti, dix contributions réparties sous les chefs suivants : Kratos, Plethos, Episteme, Nomos, Basileia, Arete.

- Victor Cousin, Platon. Textes réunis et présentés par Christiane Mauve, Michel Narcy, Renzo Ragghianti, Patrice Vermeren, Paris, Librairie philosophique J. Vrin, 2016 (Bibliothèque d'histoire de la philosophie), ISBN 978-2-7116-2730-1.

C'est à Victor Cousin (1792-1867) que l'on doit la première traduction française intégrale du corpus platonicien, publiée entre 1822 et 1840 . Sont réunis dans ce volume les «Arguments philosophiques » dont il a accompagné les dialogues contenus dans les sept premiers volumes (sur treize) de la série et deux cours sur Platon professés à l'École Normale en 1835 , restés jusqu'ici inédits.

La traduction de Cousin a fait autorité jusque dans la première moitié du $\mathrm{xx}^{\mathrm{c}}$ siècle. Elle a favorisé l'étude de Platon à un point jamais atteint jusque-là, et qui n'a pas diminué depuis : c'est elle qui a donné à Platon la place qu'il tient encore dans l'enseignement et le débat philosophique français. Étroitement liés au spiritualisme de Cousin, les commentaires dont il a assorti sa traduction ont agi comme un véhicule de cette philosophie avec d'autant plus d'efficacité que Platon n'a pas subi la désaffection dont fut frappé Cousin lui-même. C'est pourquoi ces commentaires ne se ramènent pas à un pan oublié de l'histoire des études platoniciennes en France, mais ont concouru à la constitution de la philosophie française.

-Alain Le Boulluec, Éric Junod (dir.), Anthologie des théologiens de l'Antiquité, Paris, 
Les Éditions du Cerf, 2016 (La Théologie : une anthologie), ISBN : 978-2-204-11155-3.

Présentation sous forme d'études successives rédigées par des spécialistes d'exemples significatifs de constructions doctrinales dans l'Antiquité chrétienne. La présentation par chapitres suit à peu près l'ordre chronologique des débats théologiques, en associant notices détaillées et larges extraits traduits des textes originaux; elle permet de comprendre l'élaboration progressive de la théologie chrétienne du $\mathrm{II}^{\mathrm{c}}$ au $\mathrm{V}^{\mathrm{e}}$ siècle.

Échanges de revues

- Elenchos. Rivista di Studi sulpensiero antico

36,2015 , Fasc. 2

37, 2016, Fasc. $1-2$

- Rhizomata. A Journal for Ancient Philosophy and Science

3,2015, n. 2 